\title{
Análise de atrativos químicos na coleta de flebotomineos em uma área de mata atlântica da cidade de Recife, Pernambuco, Brasil
}

\author{
Dílvia Ferreira Silva ${ }^{1}$, Simão Dias Vasconcelos ${ }^{2} \&$ Álvaro Eduardo Eiras ${ }^{3}$
}

1. Centro Universitário Maurício de Nassau. Rua Guilherme Pinto 114, Graças, Recife, PE -Brasil. Email para contato: dilvia-fs@hotmail.com

2. Departamento de Zoologia, Centro de Ciências Biológicas, Universidade Federal de Pernambuco. Av. Prof. Moraes Rego, s.n ${ }^{\circ}$, Recife - PE, 50670-420, Brasil. E-mail : simao@ufpe.br

3. Universidade Federal de Minas Gerais, Instituto de Ciências Biológicas, Departamento de Parasitologia. Av. Antônio Carlos 6627 Pampulha - Belo Horizonte, MG - Brasil. Email: alvaro@icb.ufmg.br

Recebido em : 10/01/2013. Aceito em: 18/02/2013.

\section{RESUMO}

Os flebotomineos constituem um grupo primitivo de insetos da região Neotropical que habitam áreas florestais e extraflorestais, existindo algumas espécies que vivem uma íntima associação com o homem e animais domésticos. Foi analisado o comportamento dos flebotomineos em relação a compostos químicos utilizados em armadilhas de luz. O experimento foi realizado nos meses de Novembro e Dezembro de 2000 na Mata de Dois Irmãos em Recife, onde foram testados o octenol, o $\mathrm{CO}_{2}$ e uma isca animal em diferentes combinações. Foram capturados 419 flebotomineos pertencentes ao gênero Lutzomyia: Lu. umbratilis, Lu. evandroi e L. furcata. A combinação octenol $+\mathrm{CO}_{2}$ atraiu o maior número de insetos. $\mathrm{O}$ resultado deste trabalho mostra a eficiência da utilização dos atrativos químicos nas armadilhas luminosas de capturas dos insetos.

Palavras-chave: Dióxido de carbono, Controle de Insetos vetores, Octenol.

\section{ABSTRACT}

The sandflies are a primitive group of insects Neotropical that inhabit forest and extraforest areas, there are some species that live in a close association with humans and domestic animals. Was analyzed the behavior of sandflies regarding chemical used in light traps. The experiment was conducted in November and December 2000 in the forest of Dois Irmãos in Recife, where they were tested the Octenol, $\mathrm{CO}_{2}$ and a bait animal in different combinations. Were caught 419 sandflies belonging to the genus Lutzomyia: Lu. umbratilis, Lu. evandroi and Lu. furcata. The combination octenol $+\mathrm{CO}_{2}$ has attracted the largest number of insects. The result of this study shows the efficiency of the use of chemical attractive in light traps to catch insects.

Key words: Carbon dioxide, Insects vetors Control,Octenol.

\section{INTRODUÇÃO}

As doenças transmitidas por insetos nos trópicos são de incalculável importância para a saúde pública global, com milhões de pessoas infectadas ou em risco de infecção (McCall \& Cameron 1995). Insetos hematófagos possuem diferentes hospedeiros, e os componentes do odor destes hospedeiros foram selecionados para obter resposta anemotáxica positiva (Takken \& Kline 1989). Dependendo do estado fisiológico do inseto, os mesmos odores podem induzir diferentes respostas do comportamento (Dougherty \& Hamilton 1993).

Com base nas observações do comportamento de diversas espécies de insetos em relação à atração pelo odor, foi possível isolar substâncias existentes na transpiração de animais que atraem insetos hematófagos em busca de repasto sangüíneo. Dentre os compostos encontrados destacam-se 1-octen-3- ol (octenol) e o dióxido de carbono $\left(\mathrm{CO}_{2}\right)$ (Atwood \& Meisch 1993; Dougherty et al 1993).

O dióxido de carbono figura como importante elemento que emana no ar, e foi testado para demonstrar a ativação do comportamento de procura do hospedeiro (Hamilton \& Ramsoondar 1994). É reconhecida a alta atratividade exercida pelo $\mathrm{CO}_{2}$ nos insetos hematófagos, considerando-se que é a maneira pela qual seus hospedeiros são identificados (Service, 1993).

Takken \& Kline (1989) e Kline et al (1990a) demonstraram que 1-octen-3-ol (octenol) foi um grande atrativo para Dipteros, incluindo Culicidae. Vale \& Hall (1985) em estudos de campo sobre a atração da mosca tse-tse, isolaram o 1-octen-3-ol (octenol) da respiração do boi mostrando que o seu uso pode aumentar o número de moscas próximas ao local onde ocorre a liberação deste composto. Kline et al 
(1990b) verificaram que a combinação do $\mathrm{CO}_{2}$ com o octenol aumentava significativamente o número de Aedes taeniorhynchus Wiedemann, 1821, tornando-o um atrativo mais poderoso do que outro composto químico sozinho.

Como os flebotomíneos, insetos hematófagos que apresentam grande importância médica por possuírem espécies vetores da leishmaniose, mostraram-se atraídos pelo odor de iscas tanto animais quanto humanas (Azevedo \& Rangel 1991; Nigam \& Ward 1991; Hamilton \& Ramsoondar 1994; Quinnell et al. 1992), faz-se necessário elucidar os diversos aspectos da comunicação entre esses insetos e seus hospedeiros, identificandose os compostos químicos envolvidos nesta interação.

Este estudo foi conduzido para avaliar a ação do dióxido de carbono e octenol, em diferentes concentrações, na captura de flebotomíneos, buscando contribuir com as ações de monitoramento e controle das populações desses de insetos de importância médica.

\section{MATERIAL E MÉTODOS}

As capturas de flebotomíneos foram realizadas nos meses de Novembro e Dezembro de 2000. A área escolhida foi a Mata de Dois Irmãos (34 $56^{\prime} 00^{\prime}$ W, $8^{\circ} 00^{\prime} 00^{\prime}$ ' S), situada no município de Recife. Foram utilizadas armadilhas luminosas (CDC) instaladas a $60 \mathrm{~m}$ da borda da mata, a uma distância de $100 \mathrm{~m}$ entre as armadilhas, e a uma altura do solo de $1 \mathrm{~m}$. As armadilhas permaneceram na mata durante 12 horas, das $17 \mathrm{~h}$ às $5 \mathrm{~h}$.

Para a realização dos experimentos, as armadilhas foram iscadas com compostos químicos e uma isca viva (camundongo). Os compostos utilizados foram o dióxido de carbono $\left(\mathrm{CO}_{2}\right)$ e o 1-octen-3-ol (octenol) em diferentes concentrações. $\mathrm{O}$ trabalho foi dividido em 3 experimentos, onde foram testados 5 tratamentos com 8 repetições de cada, com intervalos de 2-3 dias.

No primeiro experimento avaliou-se a atratividade do octenol com níveis de evaporação diferentes. Os tratamentos foram os seguintes: I. controle (luz); II. luz + octenol com taxa de liberação baixa (1mg/h); III. luz + octenol com taxa de liberação média $(15 \mathrm{mg} / \mathrm{h})$; IV. luz + octenol com taxa de liberação alta (30 $\mathrm{mg} / \mathrm{h}$ ) e V. luz + isca viva (camundongo Mus musculus).
Para permitir níveis diferentes de liberação, o octenol foi acondicionado em vidros de $1,5 \mathrm{ml}$ com septos e neles existiam barbantes com diferentes tamanhos. Todas as taxas de evaporação do octenol foram previamente quantificadas no Laboratório de Culicideos do Instituto de Ciências Biológicas da Universidade Federal de Minas Gerais (ICBUFMG).

No segundo experimento avaliou-se as diferentes concentrações do dióxido de carbono $\left(\mathrm{CO}_{2}\right)$, com os seguintes tratamentos: I. controle (luz); II. luz $+\mathrm{CO}_{2}$ com baixa concentração (100ml); III. luz $+\mathrm{CO}_{2}$ com média concentração $(250 \mathrm{ml})$; IV. luz $+\mathrm{CO}_{2}$ com alta concentração $(1.000 \mathrm{ml}) ; \mathrm{V}$. luz + isca viva (camundongo Mus musculus Linnaeus, 1758). Para a liberação do $\mathrm{CO}_{2}$, utilizou-se uma solução de fermento de pão, à base de Saccharomyces cerevisae. A mistura foi preparada no momento de instalação das armadilhas e colocada em garrafas plásticas de refrigerante com furos na extremidade superior, próxima à tampa.

$\mathrm{O}$ terceiro experimento utilizou cinco tratamentos com octenol $+\mathrm{CO}_{2}$ : I. controle (luz); II. luz + octenol com taxa de liberação alta $(30 \mathrm{mg} / \mathrm{h})$; III. luz $+\mathrm{CO}_{2}$ com média concentração $(250 \mathrm{ml})$; IV. luz + combinação $\mathrm{CO}_{2}(250 \mathrm{ml})$ e octenol $(30 \mathrm{mg} / \mathrm{h}) ; \mathrm{V}$. luz + isca viva (camundongo).

Os insetos coletados foram colocados em recipientes de vidro contendo álcool $70 \%$ e transferidos para o Laboratório de Invertebrados Terrestres do Departamento de Zoologia da Universidade Federal de Pernambuco - (CCB - UFPE), onde foi realizada a identificação, utilizando as chaves taxonômicas propostas por Ryan (1986) e Young \& Duncan (1994).

Nos três experimentos, comparou-se o número médio de indivíduos capturados nos diferentes tratamentos através de ANOVA não paramétrica (Kruskal-Wallis), interpretando todos os resultados com nível de significância $\mathrm{P}$ $<0,05$. As análises foram realizadas com $\mathrm{o}$ auxílio do Programa Bio-Estat 2.0 (2000).

\section{RESULTADOS}

Foi capturado um total de 875 insetos, dos quais 419 eram flebotomíneos, distribuídos entre três espécies do gênero Lutzomyia França, 1924: Lutzomyia umbratilis Ward \& Ready , 1972, L. evandroi Costa Lima \& Antunes, 1936 
e L. furcata Mangabeira, 1941. O percentual de fêmeas capturadas $(52,6 \%)$ foi levemente superior ao número de machos $(47,4 \%)$.

No primeiro experimento, observou-se diferença significativa entre os tratamentos $(\mathrm{H}=$ 17.3753, $\mathrm{P}<0,05)$. O octenol com alto nível de evaporação atraiu maior número de insetos, apresentou $\mathrm{m}=5,6$ insetos e $\mathrm{SD} \pm 1,85$. Para os demais tratamentos a média e o desvio padrão foram os seguintes: controle $\mathrm{m}=3,2$ insetos $\mathrm{e}$ $\mathrm{SD} \pm 1,83$; octenol com baixo nível de evaporação $\mathrm{m}=2,9$ e $\mathrm{SD} \pm 1,25$; octenol com médio nível de evaporação $\mathrm{m}=2,5$ e $\mathrm{SD} \pm$ 1,07; e isca animal (camundongo) $\mathrm{m}=5,0 \mathrm{e}$ $\mathrm{SD} \pm 1,69$. Lutzomyia evandroi demonstrou ser a mais atraída pelo octenol com alto nível de evaporação.

No segundo experimento, também foram observadas diferenças no número de indivíduos capturados de acordo com os atrativos utilizados $(\mathrm{H}=19.6479, \mathrm{P}<0,05) . \mathrm{O} \mathrm{CO}_{2}$ com concentração média $(250 \mathrm{ml})$ atraiu maior número de insetos, $c o m m=6,0$ e $\mathrm{SD} \pm 2,00$. A média e desvio padrão apresentados pelos outros tratamentos foram os seguintes: controle $\mathrm{m}=2,4$ e $\mathrm{SD} \pm 0,92 ; \quad \mathrm{CO}_{2} \quad$ com baixa concentração $(100 \mathrm{ml}) \mathrm{m}=2,1$ e $\mathrm{SD} \pm 0,99$; $\mathrm{CO}_{2}$ com alta concentração $(1000 \mathrm{ml}) \mathrm{m}=1,8 \mathrm{e}$ $\mathrm{SD} \pm 0,71$; e por último a isca animal $\mathrm{m}=3,1 \mathrm{e}$ $\mathrm{SD} \pm 1,64$. A espécie mais atraída pelo $\mathrm{CO}_{2}$ com concentração média foi Lutzomyia umbratilis.

No terceiro experimento, onde diferentes compostos químicos foram testados, a combinação de $\mathrm{CO}_{2}$ e octenol apresentou maior atratividade quando comparado aos outros tratamentos, com $\mathrm{m}=5,2$ e $\mathrm{SD} \pm 1,67 \quad(\mathrm{H}=$ 15.0692, $\mathrm{P}<0,05)$. Para os outros tratamentos a média e o desvio padrão foram os seguintes: controle $\mathrm{m}=3,0$ e $\mathrm{SD} \pm 1,31 ; \mathrm{CO} 2$ com concentração média $(250 \mathrm{ml}) \mathrm{m}=3,8$ e $\mathrm{SD} \pm 1,28$; octenol com alto nível de evaporação $\mathrm{m}=3,6$ e $\mathrm{SD} \pm 1,41$; isca animal (camundongo) $\mathrm{m}=2,1$ e $\mathrm{SD} \pm 0,99$. Novamente, Lutzomyia evandroi foi à espécie com maior número de indivíduos capturados.

\section{DISCUSSÃO}

Os resultados deste trabalho mostraram que diferenças nas dosagens e nas combinações dos compostos podem influir no grau de atração de espécie de flebotomíneos.

Takken \& Kline (1989) e Kline et al (1990a; 1991) verificaram que para algumas espécies de culicideos e ceratopogonideos o octenol mostrou-se eficiente, capturando um maior número desses insetos. Porém, para Atwood \& Meisch (1993) a presença do octenol pareceu reduzir a captura de simulideos.

Em nosso experimento observamos que diferentes níveis de evaporação do octenol podem apresentar diferentes respostas quanto ao número e espécie de indivíduos capturados. $\mathrm{O}$ octenol com alto nível de evaporação mostrou-se mais eficiente, sendo observado que das três espécies de flebotomíneos coletadas em todo o experimento, Lutzomyia evandroi pareceu ser a mais atraída, com maior número de indivíduos capturados.

Service (1993) verificou que espécies como Aedes vexans Meigen, 1830, Aedes spp e Anopheles walkeri Theobald, 1901 (Diptera:Culicidae) são mais atraídas quando do aumento do nível de concentração do $\mathrm{CO}_{2}$ de $1.000 \mathrm{ml}$ para $4.000 \mathrm{ml}$. Contudo, este efeito não se mostrou significante na captura de Culiseta inormata Williston,1893; Culiseta morsitans Theobald, 1901; Culex restauans e Culex pipiens Linnaeus (Diptera: Culicidae).

Neste estudo, observou-se que o nível de concentração do $\mathrm{CO}_{2}$ também influencia na atratividade das espécies de Lutzomyia. Com nível alto de concentração $(1.000 \mathrm{ml})$, ocorreu uma diminuição significante no número de indivíduos capturados. A taxa intermediária de concentração $(250 \mathrm{ml})$ capturou maior número de Lutzomyia umbratilis, talvez por estar mais próxima daquela encontrada nos seus hospedeiros.

Takken \& Kline (1989) demonstraram que o efeito do $\mathrm{CO}_{2}$ pode ser aumentado quando combinado com o octenol. A eficiência desta combinação também foi relatada por Becker et al (1995) que coletou grande número de culicídeos (Aedes taeniorhynchus Wiedemann, 1821, Anopheles atropos Dyar e Knab,1906, Anopheles crucians Lanciani and Boyt, 1977, Culex spp, Wyeomyia mitchellii Theobald, 1905), além de espécies de Ceratopogonidae e Tabanidae.

Em nosso experimento, observou-se que a combinação do $\mathrm{CO}_{2}$ com o octenol também aumentou a eficiência dos compostos quando utilizados isoladamente. As espécies Lutzomyia umbratilis e L. evandroi demonstraram maior atratividade para esta combinação, ambas com maior número de indivíduos capturados. Foi verificado também que a utilização da isca viva 
em todos os experimentos não exerceu nenhuma interferência, observando-se que os compostos químicos utilizados apresentaram um melhor nível de atratividade para os flebotomíneos.

$\mathrm{O} \mathrm{CO}_{2}$ mostrou-se como o melhor atrativo para insetos hematófagos, possibilitando a captura de um maior número de indivíduos de várias espécies e famílias. O papel do octenol utilizado isoladamente ou combinado com o $\mathrm{CO}_{2}$ merece estudos mais aprofundados, no intuito de conhecer o seu potencial na atratividade de várias outras espécies de flebotomíneos. É necessária a continuação de pesquisas sobre a interação entre insetos do gênero Lutzomyia e os atrativos químicos, para que possam ser melhor utilizados em monitoramento da população, especialmente em áreas próximas a habitações humanas. Tais informações podem colaborar no desenvolvimento de estratégias para controle destes insetos.

\section{REFERÊNCIAS BIBLIOGRÁFICAS}

Atwood, D.W. \& Meisch, M.V. 1993. Evaluation of 1octen-3-ol and carbon dioxide as black fly (Diptera:Simuliidae) attractants in Arkansas. Journal of the American Mosquito Control Association, 9: 143-146.

Azevedo, A.C.R. \& Rangel, E.F. 1991. A study of sandfly species (Diptera:Psychodidae:Phlebotominae) in a focus of cutaneous leishmaniasis in the municipality of Baturité, Ceará, Brazil. Memórias do Instituto Oswaldo Cruz, 86:405-410.

Becker, N.; Zgomba, M.; Petric, D. \& Ludwig, M. 1995. Comparison of carbon dioxide, octenol and a hostodour as mosquito attractants in the Upper Rhine attractants. Journal of the American Mosquito Control Association, 5:311-316.

Dougherty, M.J.; Hamilton, J.G. C. \& Ward, R. D. 1993. Semiochemical Mediation of Oviposition by the Phlebotomine Sandfly Lutzomyia longipalpis. Medical and Veterinary Entomology, 7: 219-224.

Hamilton, J.G.C. \& Ramsoondar, T.M.C. 1994. Attraction of Lutzomyia longipalpis to human skin odours. Medical and Veterinary Entomology, 8: 375380.

Kline, D.L.; Takken, W.; Wood, J.R. \& Carison, D. A. 1990a. Field studies on the potential of butanone, carbon dioxide, honey extract, 1-octen-3-ol, L-lactic acid and phenols as attractants for mosquitos. Medical and Veterinary Entomology, 4: 383-391.

Kline, D.L.; Wood, J.R. \& Morris, C.D. 1990 b. Evolution of 1-octen-3-ol as in attractant for
Coquilletidia perturbans, Mansonia spp. and Culex spp. associated with phosphate mining operations. Journal of the American Mosquito Control Association, 6: 605-611.

Kline, D.L.; Wood, J.R. \& Cornell, J.A. 1991. Interactive effects of 1-octen-3-ol and carbon dioxide on mosquito (Diptera:Culicidae) surveillance and control. Journal of Medical Entomology, 28: 254258.

McCall, P.J. \& Cameron, M.M. 1995. Ovoposition Pheromones in insect Vectors. Parasitology Today, 11: 352-355.

Nigam, Y. \& R.D. Ward. 1991. Male sandfly pheromone and artificial host as atractants for female Lutzomyia longipalpis (Diptera: Culicidae). Physiological Entomology, 16:305-312.

Quinnell, R.J.; Dye, C. \& Show, J.J. 1992. Host preferences of the phlebotomine sandfly Lutzomyia longipalpis in Amazonian Brazil. Medical and Veterinary Entomology, 6:195-200.

Ryan, L. 1986. Flebótomos do estado do Pará, Brasil. Wellcome Parasitology Unit, Belém, Pará, 154p.

Service, M.W. 1993. Mosquito Ecology Field Sampling Methods. $2^{\mathrm{a}}$ edition, Chapman and Hall, 988p.

Takken, W. \& Kline, D.L. 1989. Carbon dioxide and 1octen-3-ol as mosquito attractants. Journal of the American Mosquito Control Association, 5: 311-316.

Vale, G. A. \& Hall, D. R. 1985. The use of 1-octen-3-ol, acetone and carbon dioxide to improve baits for tsetse flies, Glossina spp. (Diptera: Glossinidae). Bulletin of Entomological Research, 75: 219-232.

Young, D.G. \& Ducan, M.A. 1994. Guide to the identification and geographic distribution of Lutzomyia sand flies in México, the West Indies, central and south American (Diptera: Psychodidae). Associated Publishers American Entomological Institute, Memoirs of the American Entomological Institute, 54, 881p. 